\title{
Cirurgião-dentista na equipe de saúde da família: perfil profissional e diagnóstico de cárie
}

\section{Dentist in family health team: professional profile and carie diagnosis}

\author{
EVLA ÉRINA SÁ SOUSA' \\ Samara Azevedo Gomes Campos" \\ TATYANNE SANTOS FaLCÃOIII \\ Érick Tássio Barbosa NeVes ${ }^{I V}$ \\ Matheus França Perazzolv \\ Eliane Batista de Medeiros Serpa"
}

\begin{abstract}
RESUMO
Objetivo: verificar o perfil profissional dos cirurgiões-dentistas das Equipes de Saúde da Família (ESF's) e avaliar a adequação dos diagnósticos das lesões cariosas. Metodologia: foi realizado um estudo transversal, com 97 cirurgiões-dentistas das Unidades da Saúde da Família (USF's) da cidade de João Pessoa, Paraíba. Foi utilizado um questionário dividido em duas partes. A primeira parte, abordava o perfil profissional dos participantes, e a segunda, o registro do diagnóstico da cárie dentária para dois casos clínicos. O caso clínico I correspondeu a uma lesão cariosa inativa limitada ao esmalte, e o caso clínico II, foi referente a uma cárie dentária ativa com envolvimento pulpar. Os diagnósticos foram categorizados em adequados e inadequados de acordo com critérios pré-determinados. Os dados foram analisados por meio de estatística descritiva e inferencial uni e bivariada, pelo teste Qui-Quadrado, Exato de Fischer e coeficiente de correlação de Cramer, adotando-se $p<0,05$. Resultados: os resultados mostraram a ocorrência de formados em universidades federais (89,7\%), graduados há mais de 20 anos $(63,9 \%)$, com pós-graduação $(74,7 \%)$ e possuindo como principal local de trabalho o setor público $(84,5 \%)$. O diagnóstico foi adequado em apenas $35,0 \%$ e $40,5 \%$ para os casos clínicos I e II, respectivamente. O diagnóstico de cárie dentária apenas apresentou associação com o distrito sanitário no caso clínico I. Conclusão: poucos cirurgiões-dentistas diagnosticaram adequadamente os casos clínicos, não havendo associação com o perfil profissional.
\end{abstract}

Palavras-chave: Cárie dentária; diagnóstico; odontólogos; saúde pública; odontopediatria.

\begin{abstract}
Objective: The aim of this study was to verify the professional profile of dentists from family health strategy (ESF) and assess adequacy of caries diagnostic. Methodology: It was a cross-sectional study with 97 dentists from family health units (USF's) in Joao Pessoa city, Paraiba. The first section of the questionnaire addressed the professional profile of participants, and the second part was used in order to record dental caries diagnosis according to dentists for two given clinical cases. The first clinical case was represented by an inactive dental carious lesion limited to enamel, and the second clinical case was represented by an active dental carious lesion with pulp involvement. Diagnoses were categorized in appropriate and inappropriate according to pre-determined criteria. Data was analyzed by descriptive and inferential, uni and bivariate statistics, by chi-square test, Fisher's Exact and Cramer correlation coefficient, considering $\mathrm{p}$ $<0.05$. Results: For this study, dentists graduated from federal universities accounted for $89.7 \%$, those who
\end{abstract}

\footnotetext{
Centro Odontológico de Estudos e Pesquisas (COESP), João Pessoa/PB - Brasil Universidade Federal da Paraíba (UFPB), João Pessoa/PB - Brasil

Centro Odontológico de Estudos e Pesquisas (COESP), João Pessoa/PB - Brasil

Universidade Estadual da Paraíba (UEPB), Campina Grande/PB - Brasil

v Universidade Estadual da Paraíba (UEPB), Campina Grande/PB - Brasil

Universidade Federal da Paraíba (UFPB), João Pessoa/PB - Brasil
} 
have graduated for more than 20 years ago represented $63.9 \%$, dentists with graduate degrees accounted for $74.7 \%$ and those having as main places of work the public sector represented $84.5 \%$ of the sample. Diagnosis was appropriate for only $35.0 \%$ (first clinical case) and $40.5 \%$ (second clinical case). Diagnosis of dental caries just showed an association with health district for the first clinical case. Conclusion: Few Dentists diagnosed the proposed clinical cases properly. There was not association with professional profile.

Keywords: Dental caries; diagnosis; dentists; public health; pediatric dentistry.

\section{INTRODUÇÃo}

A cárie dentária é um problema de saúde pública, com uma prevalência aproximada de 3,9 bilhões de pessoas no mundo ${ }^{1,2}$. No Brasil, $53,4 \%$ das crianças com cinco anos já apresentaram pelo menos uma lesão cariosa, de acordo com o último levantamento nacional ${ }^{3}$. A presença da cárie dentária pode acarretar impactos negativos no bem-estar funcional, social e psicológico em crianças e familiares ${ }^{4,5}$. Portanto, um correto diagnóstico é fundamental para o tratamento eficiente da alteração bucal e para a qualidade de vida do indivíduo.

Nas últimas décadas, o padrão da cárie dentária veio sofrendo mudanças profundas, sendo encontrado um grande número de lesões iniciais, reduções das lesões cavitadas e predominância de atividade nas superfícies oclusais ${ }^{6,7}$. Diante destas mudanças, o ideal é que todos os cirurgiõesdentistas, independentemente do perfil profissional, sejam capazes de diagnosticar adequadamente a cárie dentária. No entanto, variação no diagnóstico vem sendo encontrada para a mesma lesão cariosa entre os cirurgiões-dentistas ${ }^{8,9}$. Dúvidas na detecção da cárie dentária podem resultar em falsos diagnósticos e em inúmeras possibilidades de tratamento, incluindo as condutas invasivas de caráter irreversível ${ }^{10,11}$.

Diante disto, estabelecer critérios comuns aos profissionais no momento do diagnóstico poderia minimizar este problema $8,9,11$. No serviço público de saúde, considerar critérios que levem em conta a atividade da lesão cariosa no diagnóstico, é seguir o modelo que garante uma Odontologia minimamente invasiva ${ }^{12,13}$. Muitos dos estudos que avaliaram variações dos diagnósticos de cárie dentária foram realizados em ambiente acadêmico ${ }^{9,10,11}$. Esta avaliação na Estratégia de Saúde de Família permite identificar as possíveis carências sobre $\mathrm{o}$ assunto: cirurgiões-dentistas.

Portanto, o presente estudo objetivou verificar o perfil profissional e o conhecimento dos cirurgiões-dentistas de Unidades de Saúde da Família (USF's), acerca do diagnóstico da lesão cariosa em diferentes estágios de desenvolvimento em molares decíduos.

\section{Metodologia}

Foi realizado um estudo transversal com uma amostra aleatória simples de 97 cirurgiõesdentistas que trabalhavam nas Unidades de Saúde da Família no município de João Pessoa - PB. Para a realização do sorteio dos participantes, foram obtidos os dados de distribuição dos cirurgiões-dentistas por distrito sanitário na Secretaria Municipal de Saúde.

$O$ presente estudo recebeu aprovação do Comitê de Ética em Pesquisas (CEP) do Centro de Ciências da Saúde da Universidade Federal da Paraíba, com base na Resolução 196/96 do Conselho Nacional de Saúde, sob o protocolo $n^{\circ}$ 0171. Todos os direitos dos participantes foram protegidos. Os cirurgiõesdentistas leram e assinaram o Termo de Consentimento Livre e Esclarecido para a participação na pesquisa.

Para a coleta de dados foi utilizado um formulário baseado em estudo anterior ${ }^{14}$. Este instrumento apresenta duas partes principais: a primeira é voltada para a avaliação do 
perfil profissional dos cirurgiões-dentistas, observando tipo de instituição de graduação, tempo de formado, pós-graduação e local de trabalho. Já a segunda parte, objetiva o registro do diagnóstico da cárie dentária para dois casos clínicos.

Foi realizado um estudo piloto para avaliação do entendimento do formulário por parte dos cirurgiões-dentistas, por meio do método de "validação de face" com 10\% dos participantes. Para esta finalidade, o pesquisador pediu que os entrevistados explicassem com as próprias palavras o que entenderam para cada item ${ }^{15}$. Nenhum cirurgião-dentista apresentou dúvida ou dificuldade para responder os itens do formulário. A confiabilidade teste-reteste foi determinada após um intervalo de sete dias entre a aplicação dos formulários com os mesmos indivíduos. A concordância entre os testes foi de $95 \%$. A aplicação dos questionários durou aproximadamente dez minutos. Os participantes do piloto não foram incluídos na amostra.

Os casos clínicos foram selecionados do arquivo da clínica de Odontopediatria da Universidade Federal da Paraíba. Foram selecionados dois que representassem diferentes estágios da cárie em molares decíduos. Como critérios de inclusão, a criança deveria possuir boa saúde geral, não estar sob tratamento medicamentoso e possuir peso e altura compatíveis com a idade. Além disto, a documentação deveria apresentar: ficha clínica devidamente preenchida; radiografia inicial da lesão cariosa; fotografia intra-oral; termo de autorização e consentimento assinados pelos pais permitindo o uso dos dados clínicos da criança em pesquisas. Todos os direitos da criança foram preservados.

Os cirurgiões-dentistas responderam o diagnóstico que acreditavam ser o mais apropriado com base no relato do caso, fotografia intra-oral e radiografia periapical. A resposta de cada caso foi comparada aos critérios estabelecidos por Miller ${ }^{16}$, instrumento popularmente utilizado para o diagnóstico da atividade das lesões cariosas em dentina, o que padroniza e facilita comparações entre trabalhos ${ }^{14}$.

De acordo com os critérios de Miller ${ }^{16}$, a cárie dentária inativa possui uma superfície escura, consistência dura, geralmente assintomática, progressão lenta, intermitente, a dentina adjacente esclerótica e pigmentada. Já a lesão cariosa ativa em dentina possui cor clara; é geralmente sintomática para comidas doces, ácidas e ao frio; possui camada superficial com consistência macia, é friável e coberta por uma massa necrótica; tem progressão rápida e dentina descalcificada.

Os dados foram analisados inicialmente através de estatística descritiva. O teste do Qui-Quadrado, Exato de Fischer e coeficiente de correlação de Cramer foram utilizados para testar e avaliar a associação entre os diagnósticos com as variáveis sociodemográficas e os tipos de distritos sanitários. Os dados foram organizados e analisados pelo programa de informática Statistical Package for Social Sciences (SPSS para Windows, versão 20.0, SPSS Inc, Chicago, IL, EUA). O nível de significância adotado foi $5 \%$.

\section{Relato de Caso}

\section{Caso Clínico I}

Uma menina aos 9 anos de idade, com boa higiene oral, consumia pouco carboidrato, escovava os dentes três a quatro vezes ao dia, mas não fazia uso do fio dental. Não apresentava sintomatologia dolorosa em nenhum elemento e buscou o serviço de odontologia para exame de rotina. De acordo com as Figuras 1 e 2, qual o diagnóstico da lesão para o elemento 85 ?

Diagnóstico adequado: Lesão cariosa inativa, restrita ao esmalte oclusal. 


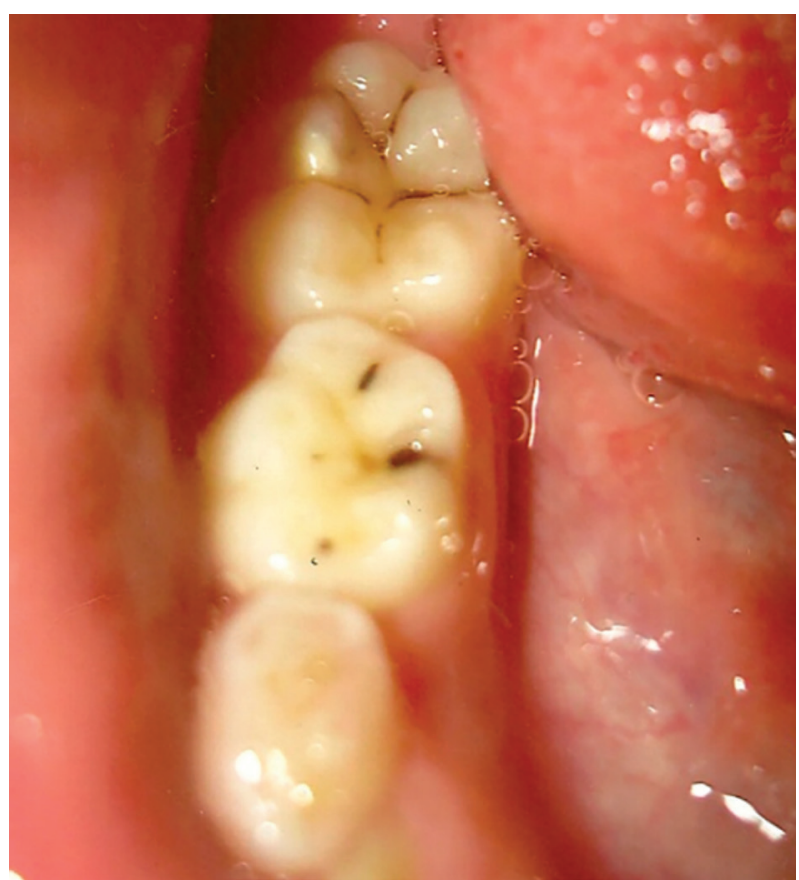

Figura 1 - Aspecto clínico da lesão cariosa do dente 85.

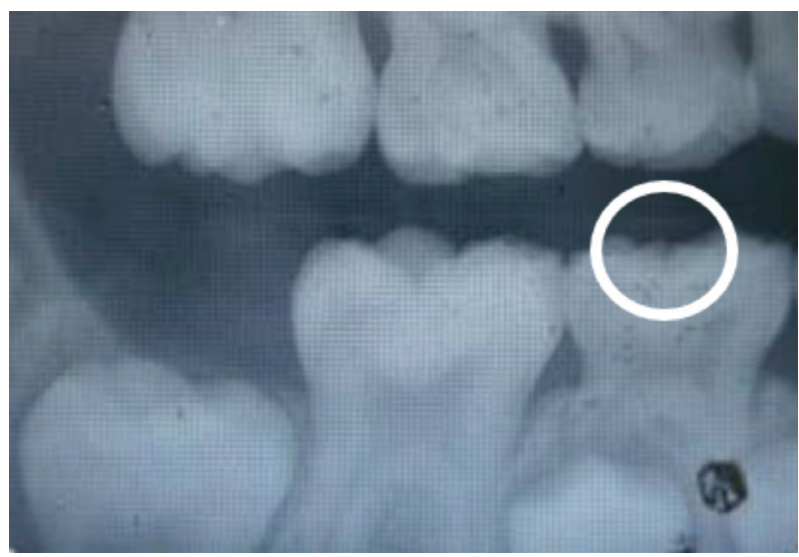

Figura 2 - Aspecto radiográfico da lesão cariosa do dente 85 .

\section{Caso Clínico II}

Um menino aos 8 anos de idade, com alto consumo de sacarose, fazia uso de mamadeira noturna. Escovava os dentes duas vezes ao dia sem supervisão do pai/responsável e não utilizava o fio dental na higienização. Relatou dor provocada ao mastigar. De acordo com as Figuras 3 e 4, qual o diagnóstico da lesão para o elemento 84 ?
Diagnóstico adequado: Lesões cariosas ativas com envolvimento pulpar.

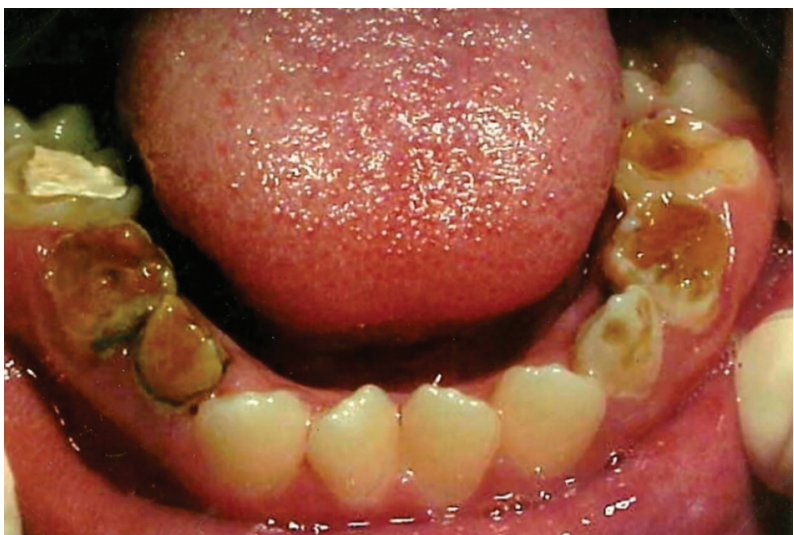

Figura 3 - Aspecto clínico da lesão cariosa do dente 84.

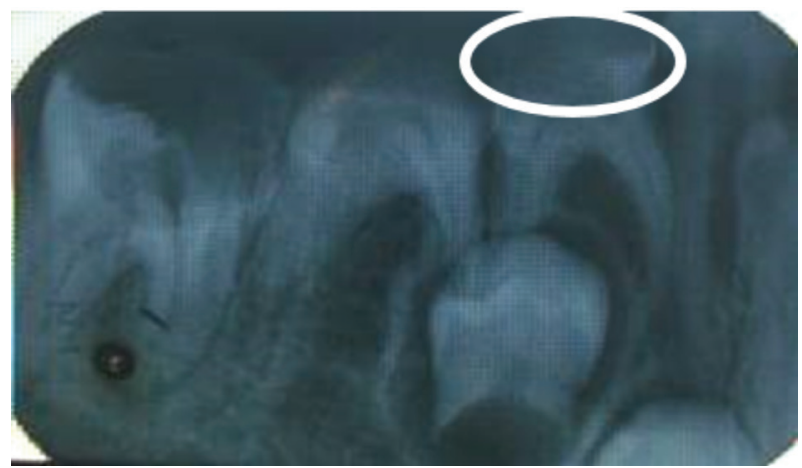

Figura 4 - Aspecto radiográfico da lesão cariosa do dente 84

\section{Resultados}

A amostra com 97 cirurgiões-dentistas do USFs de João Pessoa/PB, caracterizouse por: a) maior prevalência de formados em universidades públicas $(97,9 \%)$, b) graduados há mais de 20 anos $(63,9 \%)$, c) com pósgraduação $(74,7 \%)$ d) tendo como principal local de trabalho o setor público exclusivamente $(84,5 \%)$ (Tabela 1).

Os casos clínicos selecionados retrataram as lesões cariosas mais frequentes em molares decíduos na amostra estudada: o caso clínico I correspondia a uma lesão cariosa de fissura em esmalte inativa, e o caso clínico II, tratavase de uma lesão cariosa aguda com grande destruição coronária e envolvimento pulpar, de todos os molares decíduos. O diagnóstico adequado foi relatado por $35,0 \%$ e $40,1 \%$ dos cirurgiões-dentistas respectivamente (Tabela 2). 
Em ambos os casos, o diagnóstico inadequado foi representado principalmente pela não especificação da atividade da lesão cariosa, seguido da identificação incorreta desta atividade.

Tabela 1 - Formação profissional e local de trabalho dos cirurgiões-dentistas.

\begin{tabular}{lcc}
\hline \multicolumn{1}{c}{$\begin{array}{c}\text { Caracterização da } \\
\text { amostra }\end{array}$} & $\mathbf{N}$ & TOTAL \\
\cline { 2 - 3 } & & \\
\hline Tipo de Instituição & 87 & 89,7 \\
Federal & 08 & 8,2 \\
Estadual & 2 & 2,1 \\
Privada & & \\
Tempo de Graduação & 16 & 16,5 \\
Até 5 anos & 7 & 7,2 \\
6 a 10 anos & 12 & 12,4 \\
11 a 20 anos & 62 & 63,9 \\
> 20 anos & & \\
Pós-Graduação & 71 & 74,7 \\
Sim & 24 & 25,3 \\
Não & & 84,5 \\
Local de Trabalho & 82 & 15,5 \\
Público & 15 & \\
Público e Privado & & \\
\hline
\end{tabular}

Tabela 2 - Frequência dos diagnósticos nos casos clínicos.

\begin{tabular}{lcc}
\hline \multicolumn{1}{c}{ Diagnóstico } & \multicolumn{2}{c}{ TOTAL } \\
\cline { 2 - 3 } & $\mathbf{N}$ & \\
\hline Caso Clínico I & & \\
\hline Cárie inativa limitada ao esmalte & 34 & 35,0 \\
Cárie (sem relato da atividade) & 27 & 27,8 \\
Cárie incipiente & 09 & 9,3 \\
Cárie ativa & 18 & 18,6 \\
Mancha pré-cariosa & 09 & 9,3 \\
\hline Caso Clínico II & & \\
\hline Cárie ativa com envolvimento pulpar & 39 & 40,1 \\
Cárie (sem relato da atividade) & 21 & 21,7 \\
Cárie inativa & 17 & 17,6 \\
Cárie de mamadeira/ rampante & 15 & 15,6 \\
Cárie inicial & 01 & 1,0 \\
Erosão dentária & 01 & 1,0 \\
Pulpite associado à periodontite & 02 & 2,0 \\
Hipocalcificação / má formação dentária & 01 & 1,0 \\
\hline
\end{tabular}

A Tabela 3 refere-se à análise bivariada, onde nenhuma variável relacionada à formação profissional ou local de trabalho apresentou associação com o diagnóstico relatado pelos cirurgiões-dentistas. Os distritos sanitários foram os únicos que apresentaram relação com o diagnóstico, apenas para o caso clínico I. No entanto, o coeficiente de Cramer ( $V=0,333)$ mostrou que esta associação foi fraca.

Tabela 3 - Análise bivariada entre o diagnóstico dos casos clínicos e as variáveis independentes.

\begin{tabular}{|c|c|c|c|c|c|c|c|c|c|c|}
\hline \multirow{3}{*}{ Variáveis } & \multicolumn{5}{|c|}{$\begin{array}{c}\text { Diagnóstico } \\
\text { Caso Clínico I }\end{array}$} & \multicolumn{5}{|c|}{$\begin{array}{c}\text { Diagnóstico } \\
\text { Caso Clínico II }\end{array}$} \\
\hline & \multicolumn{2}{|c|}{ Adequado } & \multicolumn{2}{|c|}{ Inadequado } & \multirow{2}{*}{$\mathbf{P}$} & \multicolumn{2}{|c|}{ Adequado } & \multicolumn{2}{|c|}{ Inadequado } & \multirow{2}{*}{$\mathbf{P}$} \\
\hline & $\mathbf{N}$ & $\%$ & $\mathbf{N}$ & $\%$ & & $\mathbf{N}$ & $\%$ & $\mathbf{N}$ & $\%$ & \\
\hline $\begin{array}{l}\text { Tipo de instituição } \\
\text { Federal } \\
\text { Estadual } \\
\text { Privada } \\
\end{array}$ & $\begin{array}{c}31 \\
2 \\
1 \\
\end{array}$ & $\begin{array}{l}35,6 \\
22,2 \\
50,0\end{array}$ & $\begin{array}{l}56 \\
6 \\
1 \\
\end{array}$ & $\begin{array}{l}64,4 \\
77,8 \\
50,0\end{array}$ & 0,754 & $\begin{array}{l}38 \\
3 \\
2 \\
\end{array}$ & $\begin{array}{c}43,7 \\
37,5 \\
100,0 \\
\end{array}$ & $\begin{array}{c}49 \\
5 \\
0\end{array}$ & $\begin{array}{c}56,3 \\
62,5 \\
0,0 \\
\end{array}$ & 0,262 \\
\hline $\begin{array}{l}\text { Tempo de formado } \\
\text { Até } 5 \text { anos } \\
6-10 \text { anos } \\
11-20 \text { anos } \\
\text { Mais de } 20 \text { anos } \\
\end{array}$ & $\begin{array}{c}5 \\
3 \\
5 \\
21\end{array}$ & $\begin{array}{l}31,2 \\
42,8 \\
41,6 \\
33,8 \\
\end{array}$ & $\begin{array}{c}11 \\
4 \\
7 \\
41 \\
\end{array}$ & $\begin{array}{l}68,8 \\
57,2 \\
58,4 \\
66,2 \\
\end{array}$ & 0,906 & $\begin{array}{c}11 \\
3 \\
5 \\
24 \\
\end{array}$ & $\begin{array}{l}68,7 \\
42,8 \\
41,7 \\
38,7 \\
\end{array}$ & $\begin{array}{c}5 \\
4 \\
7 \\
38 \\
\end{array}$ & $\begin{array}{l}31,3 \\
57,2 \\
58.3 \\
61,3 \\
\end{array}$ & 0,195 \\
\hline $\begin{array}{l}\text { Pós-Graduação } \\
\text { Sim } \\
\text { Não }\end{array}$ & $\begin{array}{c}28 \\
6\end{array}$ & $\begin{array}{l}38,3 \\
25,0\end{array}$ & $\begin{array}{l}45 \\
18 \\
\end{array}$ & $\begin{array}{l}61,7 \\
75,0\end{array}$ & 0,234 & $\begin{array}{l}33 \\
10 \\
\end{array}$ & $\begin{array}{l}45,2 \\
41,7\end{array}$ & $\begin{array}{l}40 \\
14 \\
\end{array}$ & $\begin{array}{l}57,8 \\
58,3\end{array}$ & 0,762 \\
\hline $\begin{array}{l}\text { Local de trabalho } \\
\text { Público } \\
\text { Público e Privado }\end{array}$ & $\begin{array}{c}26 \\
8\end{array}$ & $\begin{array}{l}31,7 \\
53,3\end{array}$ & $\begin{array}{c}56 \\
7\end{array}$ & $\begin{array}{l}68,3 \\
46,7\end{array}$ & 0,107 & $\begin{array}{c}36 \\
7\end{array}$ & $\begin{array}{l}44,0 \\
46,7\end{array}$ & $\begin{array}{c}46 \\
8\end{array}$ & $\begin{array}{l}56,0 \\
53,3\end{array}$ & 0,843 \\
\hline $\begin{array}{l}\text { Distritos Sanitários } \\
\text { I } \\
\text { II } \\
\text { III } \\
\text { IV } \\
\text { V }\end{array}$ & $\begin{array}{c}2 \\
14 \\
5 \\
11 \\
2\end{array}$ & $\begin{array}{l}11,8 \\
50,0 \\
23,8 \\
50,0 \\
22,2\end{array}$ & $\begin{array}{l}15 \\
14 \\
16 \\
11 \\
7 \\
\end{array}$ & $\begin{array}{l}88,2 \\
50,0 \\
76,2 \\
50,0 \\
77,8\end{array}$ & $0,029^{*}$ & $\begin{array}{c}5 \\
15 \\
7 \\
14 \\
2 \\
\end{array}$ & $\begin{array}{l}29,4 \\
53,6 \\
33,3 \\
63,6 \\
22,2 \\
\end{array}$ & $\begin{array}{l}12 \\
13 \\
14 \\
8 \\
7\end{array}$ & $\begin{array}{l}70,6 \\
46,4 \\
66,7 \\
36,4 \\
77,8\end{array}$ & 0,071 \\
\hline
\end{tabular}




\section{Dıscussão}

Os casos clínicos I e II apresentados
aos cirurgiões-dentistas das USF's
incluíram lesões cariosas inativa e ativa,
respectivamente, em molares decíduos.
A ocorrência do diagnóstico adequado foi baixa para ambos os casos. Esta baixa concordância entre os diagnósticos pode decorrer da subjetividade dos profissionais na detecção da lesão cariosa, principalmente quanto à atividade. Tal quadro, assim como os encontrados em outros estudos, pode acarretar no sub ou sobretratamento do paciente, como também trazer impactos ao serviço de saúde s,11,17. $^{8}$.

No serviço público de saúde, um diagnóstico inadequado, em especial os falso-positivos, insere desnecessariamente o paciente em um ciclo restaurador, aumentando a demanda e o tempo de espera para acessar ao serviço 8,10,11. A solução para reduzir a subjetividade nos diagnósticos de cárie dentária é padronizar os critérios de avaliação ${ }^{8,18}$. Tradicionalmente, o índice utilizado é o proposto pela Organização Mundial da Saúde (OMS) $)^{19}$, no entanto, recebe muitas críticas por não avaliar a atividade nem as lesões iniciais de cárie, fundamentais para ações preventivas nos serviços públicos de saúde $^{20}$. Por isto, o presente estudo utilizou como método diagnóstico os indicados por Miller $^{8}$, pois considerou a atividade da lesão cariosa, importante para uma Odontologia minimamente invasiva ${ }^{12,13}$.

Em relação ao perfil profissional, os cirurgiões-dentistas das USF's do município apresentaram características semelhantes aos encontrados em outras regiões do Brasil. Comumente há uma prevalência de profissionais formados em universidades públicas e com pós-graduação, no entanto, discordâncias são encontradas entre as regiões, quanto ao tempo de formado e o local de trabalho $21,22,23,24,25$.
O diagnóstico de cárie apenas apresentou uma associação fraca para os distritos sanitários no caso clínico I. O fato do diagnóstico não estar relacionado com nenhuma variável da formação profissional avaliada pode sugerir que a baixa prevalência dos diagnósticos adequados decorra de um outro fator, relacionado à formação dos cirurgiõesdentistas. Provavelmente, sofreu influência da metodologia de ensino empregada nas instituições refletindo no conhecimento dos profissionais e consequentemente sobre a forma que encaram o processo de saúdedoença ${ }^{11,26}$.

Nos dois casos clínicos, cerca de um quarto dos participantes não relataram a atividade da lesão, o que sugere a utilização dos critérios da $\mathrm{OMS}^{19}$ como referência por estes profissionais. Por outro lado, houve cirurgiõesdentistas que diagnosticaram a atividade das lesões, porém inadequadamente, o que também sugere uma deficiência no domínio do assunto.

Portanto, diante da baixa taxa de diagnósticos adequados, é necessário que haja uma padronização/atualização dos critérios para o diagnóstico de cárie utilizados pelas ESF's. A padronização facilitaria a comunicação entre diferentes profissionais e refletiria sobre o padrão epidemiológico da população ${ }^{8}$. A garantia deste objetivo pode ocorrer por meio da educação continuada dos cirurgiões-dentistas, visto que representa uma forma de atualizar e preencher as lacunas no conhecimento de assuntos específicos ${ }^{27,28}$.

Pelas características do desenho deste estudo transversal, não foi possível estabelecer relações de causa/efeito nem realizar extrapolações, considerando que não houve validação externa. Mesmo assim, ficou evidente que a falta de uma padronização dos critérios de diagnóstico de cárie dentária nas ESF's representa um problema a ser solucionado nos serviços públicos de saúde. 


\section{Conclusão}

O perfil dos cirurgiões-dentistas das ESF's mostrou alta experiência profissional. No entanto, a ocorrência do correto diagnóstico foi baixa nos dois casos clínicos, visto que poucos profissionais basearam o diagnóstico no padrão de atividade da lesão cariosa de forma adequada. Quanto à associação, não foi encontrada relação entre o perfil profissional e os diagnósticos das lesões cariosas.

\section{REFERÊNCIAS}

1. Bagramian RA, Garcia-Godoy F, Volpe AR. The global increase in dental caries. A pending public health crisis. Am J Dent. 2009; 22(1): 3-8.

2. Richards $D$. Oral diseases affect some 3.9 billion people. Evid Based Dent. 2013; 14(2): 35.

3. Brasil. Ministério da Saúde. SB Brasil 2010, Pesquisa Nacional de Saúde Bucal: Resultados Principais. 2012. [acesso em 18 nov. 2015]. Disponível em: http://bvsms.saude. gov.br/bvs/publicacoes/pesquisa_nacional_ saude_bucal.pdf.

4. Scarpelli AC, Paiva SM, Viegas CM, Carvalho AC, Ferreira FM, Pordeus IA. Oral healthrelated quality of life among Brazilian preschool children. Community Dent Oral Epidemiol. 2013; 41(4): 336-44.

5. Gomes MC, Pinto-Sarmento TC, Costa EM, Martins CC, Granville-Garcia AF, Paiva SM. Impact of oral health conditions on the quality of life of preschool children and their families: a cross-sectional study. Health Qual Life Outcomes. 2014; 12:55.

6. Jain SK, Pushpanjali K, Reddy SK, Gaikwad R, Deolia S. Comparison of different caries diagnostic thresholds under epidemiological and clinical settings among 7-15 year old school children from Bangalore city. J Int Soc Prev Community Dent. 2013; 3(2): 85-91.

7. Silva RP, Assaf AV, Ambrosano GMB, Mialhe FL, Meneghim MC, Pereira AC. Different methods of dental caries diagnosis in an epidemiological setting. Braz J Oral Sci. 2015; 14(1): 78-83.
8. Mialhe FL, Silva RP, Ambrosano GMB, Pereira AC, Ferreira AC. Occlusal caries detection and its treatment by public health service dentists. RFO. 2007; 12(3): 29-34.

9. Bobrowski R, Schneider M. Divergence between diagnostic healthy, biological sealing and occlusal caries in enamel or enamel and dentin, carried out by academics and dental professionals. Stomatos. 2011; 17(32): 43-54.

10. Coelho LT, Silveira ADS, Lima KC, Pinheiro IVA. Oclusal decay without cavitation: divergency on the terapeutic decision. Odontol Clín-Cientif. 2007; 6(1): 39-43.

11. Silva RP, Ferreira AC, Mialhe FL, Ambrosano GMB. Variabilidade na detecção e tratamento da cárie dentária in vitro por acadêmicos: a importância do processo ensino-aprendizagem. UFES Rev Odontol. 2007; 9(1): 33-40.

12. Mount GJ, Ngo H. Minimal intervention: a new concept for operative dentistry. Quintessence Int. 2000; 31(8): 527-33.

13. Tyas MJ, Anusavice KJ, Frencken JE, Mount GJ. Minimal intervention dentistry--a review. FDI Commission Project 1-97. Int Dent J. 2000; 50(1): 1-12.

14. Medeiros EB, Rosenblatt A, Rodrigues MJ. Diagnóstico das lesões cariosas ativa e inativa em dentina de molares decíduos. Odontol Soc. 2007; 7(1): 30-8.

15. Frankfort-Nachimias $C$, Nachimias D. Research methods in the social sciences. London: Arnold; 1992.

16. Miller WA. Layering in dentin caries as demonstrated by localization of dyes. Illinois. Tese (Mestrado) - University of Illinois; 1959.

17. Coelho LT, Silveira ADS, Lima KC, Pinheiro IVA. Oclusal decay without cavitation: divergency on the terapeutic decision. Odontol Clín.-Cientif. 2007; 6(1): 39-43.

18. Braga MM, Mendes FM, Ekstrand KR. Detection activity assessment and diagnosis of dental caries lesions. Dent Clin North Am. 2010; 54(3): 479-93.

19. Organização Munidial da Saúde. Oral Health Surveys: Basic methods. 4th ed. Geneva: Switzerland; 1997.

20. Honkala E, Runnel R, Honkala S, Olak J, Vahlberg T, Saag M, et al. Measuring Dental 
Caries in the Mixed Dentition by ICDAS. Int $\mathbf{J}$ Dent. 2011; 2011:150424

21. Maciel CF, Barcellos LA, Miotto MHMB. Perfil dos Cirurgiões-Dentistas do Programa de Saúde da Família da Grande Vitória - parte I. UFES Rev Odontol. 2006; 8(3): 31-7.

22. Vilarinho SMM, Mendes RF, Prado-Júnior, RR. Profile of the dentists that participate in the family health program of Teresina (PI). Rev odonto Ciênc. 2007; 22(55): 49-54.

23. Rodrigues AAAO, Gallotti AP, Pena SFA, Ledo CAS. Dental health in Feira de Santana (BA) Family Health Program: the surgeon dentist profile. RBSP. 2009; 33(4): 582-94.

24. Lenzi TL, Rocha RO, Dotto PP, Raggio DP. Profile of the dentists members of Family Health Program in a city in the South part of Brazil. $\mathbf{J}$ Health Sci Inst. 2010; 28(2): 121-4.
25. Bottan ER, Vitoretti AJ, Uriarte-Neto M. Professional profile of the dental surgeon working in the public service: the views of a group of dental surgeons. Rev Bras Ciên Saúde. 2014; 12(40): 42-7.

26. Martins IM, Pereira PZ, De-Carli AD. EvidenceBased Cariology and the Teaching-Learning Process. Rev Bras Educ Med. 2015; 39(1): 50-9.

27. Sabadin LF, Lodi CS. Practical organization proposals for the oral health actions in the family public health care center. A literature review. Rev Fac Odontol Lins. 2013; 23(1): 26-36.

28. Campos L, Reiser LM, Bottan ER, Silveira EG. Knowledge and Attitudes about Oral Health: Study with Community Health Workers From Itajaí (SC). Rev Fac Odontol Lins. 2015; 25(1): 11-16.

Submetido em: 10-5-2016

Aceito em: 11-8-2016 